\title{
DETC2007-35188
}

\section{A PROPOSED METHOD TO PRESERVE KNOWLEDGE AND INFORMATION BY USE OF KNOWLEDGE ENABLED ENGINEERING}

\author{
Stefan Sandberg \\ Division of Computer Aided Design \\ Luleå University of Technology \\ SE-971 87 Luleå, Sweden \\ Stefan.Sandberg@Itu.se
}

\author{
Mats Näsström \\ Division of Computer Aided Design \\ Luleå University of Technology \\ SE-971 87 Luleå, Sweden \\ Mats.Nasstrom@Itu.se
}

\begin{abstract}
Today's automotive industry produces more new car models in a shorter time than ever before. Every car model comes in many different versions regarding number of doors, engine, transmission etc while being built on a platform strategy. This leads to a lot of different knowledge and information that needs to be tracked for the different components in addition to other information e.g. what function does design features have, why is this radius not smaller etc. Volvo Car Corporation (VCC) is in need of an effective method to save and present all this knowledge and information today. This paper describes a new method, to gather and save knowledge and information about car body parts also called body-in white, which was implemented in a demonstrator and tested and evaluated on VCC.
\end{abstract}

\section{INTRODUCTION}

Many companies are bought and included in larger companies. The Swedish automotive manufacturer Volvo Car Corporation (VCC), where this research has been carried out, is owned by Ford Motor Company. In these enterprises a lot of body-in-white parts, see figure 1 , are shared and they build common platforms, e.g. a front, floor etc, that will be used by other companies within the enterprise. Every company uses so called carry-overs as well, meaning that many new models share components with an earlier car model or platform. For instance, a component in a new car model will be the exact same as in an already released car model. By using carry-overs they can shorten lead times and save money since they don't have to develop any new design or production tools etc. When companies use carry-overs or if component requirements change later in a project it can cause problems. Problems can be related to the knowledge concerning why a certain design or feature is created the way it is and that the engineers creating the new car or platform do not know the reason behind the design or feature. Hence the engineers do not feel confident to make changes to the component since they cannot make a well informed decision e.g. what happens if we change this radius? Why is the radius this size? If the designer doesn't have sufficient knowledge about the specific component it may lead to the wrong decision that might lead to higher cost, weight etc.

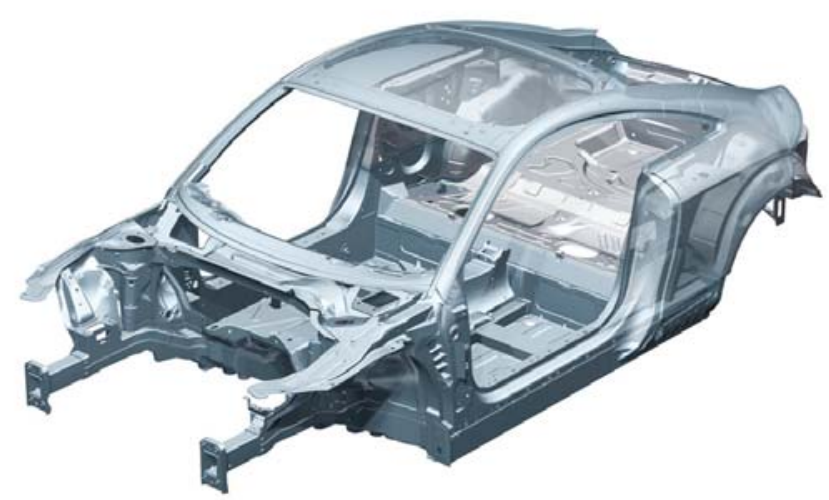

Figure 1 Picture of car body parts generally spoken of as the body-in-white.

A large problem is all this knowledge and information about these different components, and all their different versions and configurations that needs to be saved and presented in an effective way. How about the knowledge about 
why the component ended in that particular design, also called design rationale? Why is this feature located here, what's the function of this hole and why is this radius not smaller? Discussions with other Swedish manufacturing companies reveal that this "design history" issue is a general problem today. The purpose of this study is to highlight a new method by the development of a demonstrator which capture knowledge and information i.e., design rationale. By doing this, the design rationale will be saved for use later on.

\section{SITUATION OF TODAY}

The idea for this new method came after literature research and many discussions with designers on VCC and the need became more and more clear. This knowledge on VCC is today saved in fragments on a 2D-drawing or exists only in the head of the designer. Half of the designers work in the CAD-program CATIAv4 today and half in CATIAv5 but all new design are made in the latter. VCC is also on the way to only work in $3 \mathrm{D}$ with 3D-PMI (Product Manufacturing Information) that follows the standard ASME Y14.41 [1], replacing the 2Ddrawings. PMI consists for example of:

- Material, Material thickness and material direction.

- Holes, including centre line.

- GD\&T (General Dimensioning and Tolerances)

- Mating surfaces

- Drawing information (drawing head, text annotations such as general demands and annotations of changes)

It's also a way to keep what you can call the core knowledge in the company since it's not uncommon that the designer is hired only to work on one specific project and there is a possibility that the designer is not with the company when answers about the design rationale must be found.

Design review meetings are taking place every week or two at VCC where the designer gets feedback on his design from experts in materials, simulations and manufacturing etc. Today they meet in a room usually outfitted with two screens. On one screen they show a 3D CAD model and on the other screen they have a PowerPoint document. During the meeting they can navigate in the 3D model to show specific areas of interest and when they make comments and notes they do it in the PowerPoint document. The PowerPoint document contains 2D pictures of the component and comments are made with colored text and arrows. Usually they use green, yellow and red text as a status indicator of the comments.

A component can be used as a carry-over or just as a reference for the designer to look at to get ideas for the new component in a new project. In Figure 2, the left side shows a car that's already produced and released to the market. The right side shows a car to be designed. The two models share part $\mathrm{A}$ as a carry-over, but parts $\mathrm{D}$ and $\mathrm{E}$ requires a totally new design or some redesign, e.g., $\mathrm{D}$ is similar to $\mathrm{B}$ and hence $\mathrm{B}$ can be used as a reference model whilst E doesn't share any requirements with $\mathrm{C}$ and will be designed from scratch. In the beginning of the development process the level of abstraction is high. It is important to decrease the level of abstraction and increase the knowledge about different solutions being made as early as possible in the product development process, see figure 3. By increasing the level of knowledge the level of abstraction is reduced allowing better decisions [2].

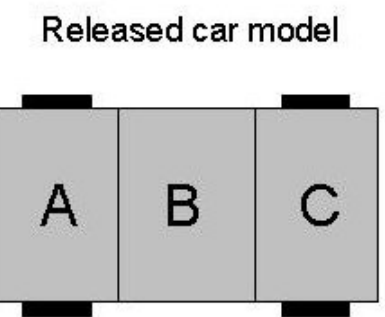

New car model

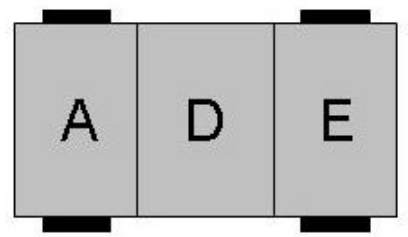

Figure 2 A simplified picture of two cars divided into only three parts.
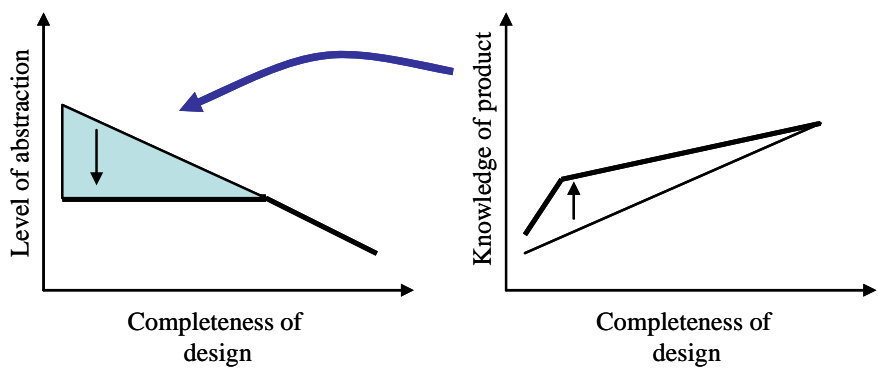

Figure 3 Representation of a decreased level of abstraction and increased knowledge with the use of knowledge based methods.

\section{KNOWLEDGE ENABLED ENGINEERING}

Knowledge Enabled Engineering (KEE) [3] is an approach to design, which enables the design process to be guided by relevant and verifiable knowledge as needed. Information from design rationale, simulation results, physical tests and aftermarket research on earlier similar products provides the basis for the knowledge in use. A design process driven by knowledge is likely to prevent costly design changes in later stages. Furthermore, it might even be impossible to compensate or correct the shortcomings of a poor design concept later on. Hence, KEE represents the combination of the logics of the design object and the actual design process used to create the object, and together this allows for the generation of design solutions (e.g. geometries and more) 


\subsection{Knowledge Based Engineering}

Included in the KEE approach is the support of Knowledge Based Engineering (KBE) methods, which provide possibilities to formalize and preserve knowledge. KBE springs from Knowledge Based Systems but with a tighter connection to geometry modeling. An example of knowledge captured and formalized in a KBE application is how to make a FEsimulation of a beam section in a car or to calculate the stiffness of a joint [4]. Another example of captured and formalized knowledge is how hardening due to sheet-metal forming will affect the crashworthiness of a beam [5]. Many other successful industry applications can also be mentioned according to $[3,6]$.

\section{CASE STUDY}

The design of the demonstrator along with which knowledge and information that was needed to be saved was decided together with a reference group at VCC. The reference group consisted of three senior car body designers. The first step was a Future Workshop [7, 8], held at VCC with the reference group. A Future Workshop is divided into three phases:

- The Critique phase is designed to draw out specific issues and problems about current work practice

- The Fantasy phase allows the participants to imagine "what if" the workplace could be different

- The Implementation phase focuses on what changes that is realistic to accomplish and what resources would be needed
After the Future Workshop the data, knowledge and information that was suitable to save, was sorted and graded. This sorting and grading process was done together with the reference group through e-mails and telephone meetings.

\section{DEMONSTRATOR}

After the sorting and grading of the knowledge and information was done the demonstrator was built in CATIAv5 with help from Volvo Information Technology (Volvo IT). CATIAv5 was chosen since it is the CAD environment that will be used in the future and the designers wouldn't have to learn a new application which was positive according to them. During the building of the demonstrator discussions was held regularly with a reference group consisting of four designers at VCC to get constant feedback of the progress. The demonstrator shows how knowledge and information can be saved directly in the model with the help of 3D-annotations as plain text, headings with more hidden text displayable via a click on the heading, hyperlinks to URL's and external documents, see figure 4.

Figure 5 shows an example of extended knowledge about why the tolerance of a flange is not company standard, i.e., representing what knowledge and information that can be saved, in this case it tells why a tolerance isn't company standard.

Another reason to why the demonstrator was built in CATIAv5 is that it has the option to export the model as .jt-file that can be viewed in software like TeamCenter and VisMockup, since this allows for easy collaboration with personnel that do not have their own CATIAv5 workstation.

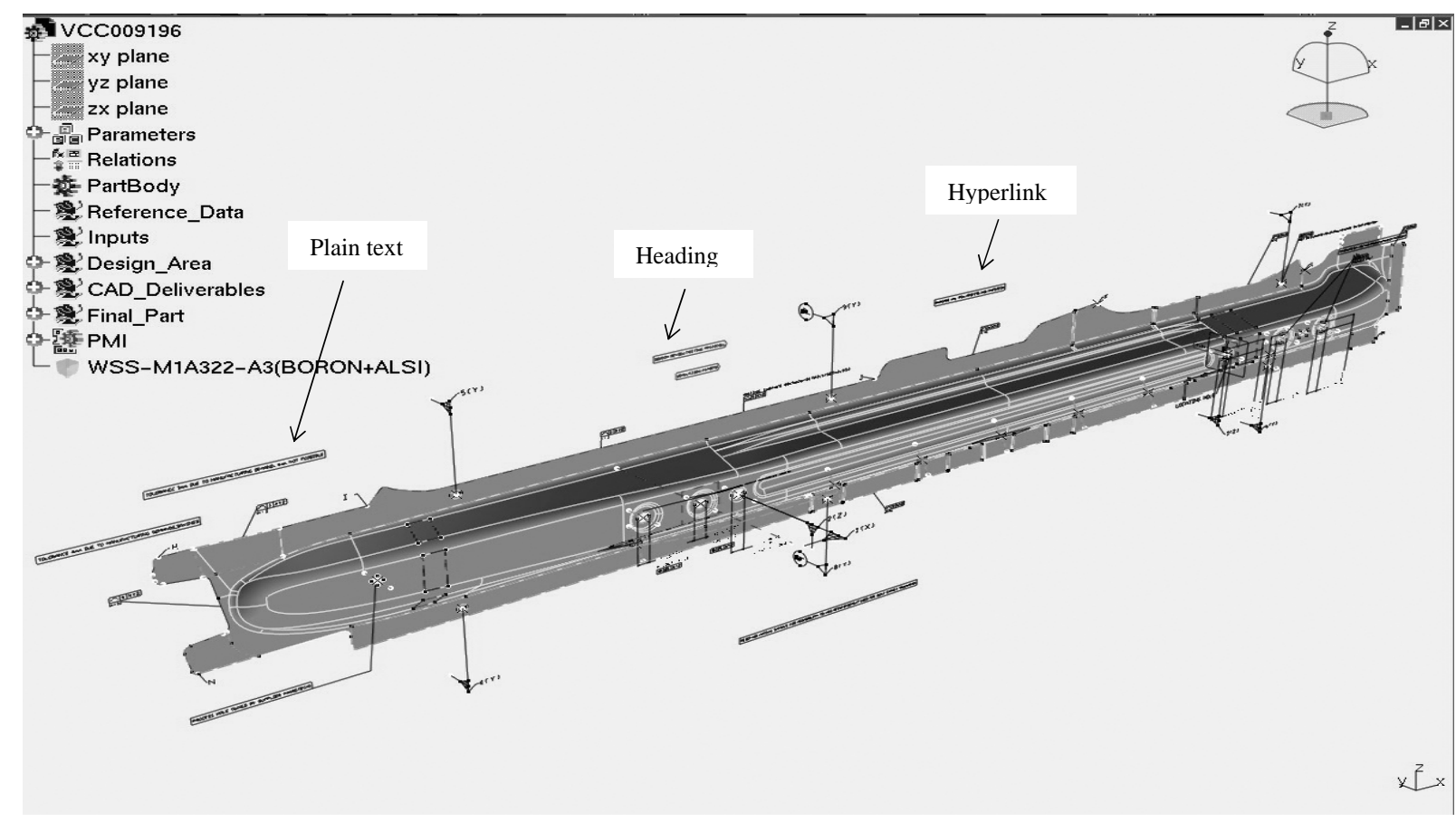

Figure 4 3D-model with both 3D-PMI and design rationale directly in the model. 


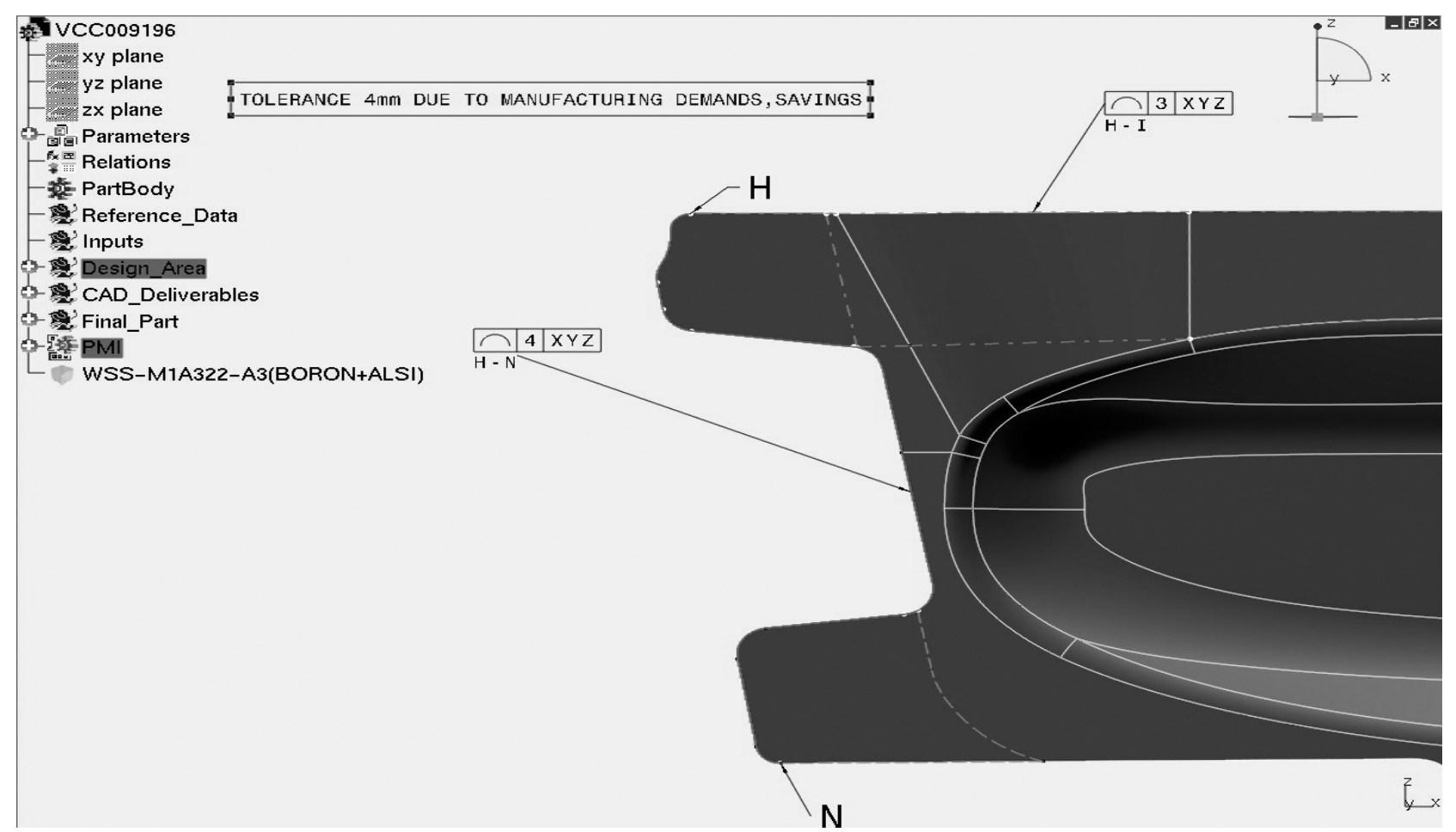

Figure 5 Example of what knowledge and information that can be saved.

By saving the knowledge and information this way it secures that it will always follow with the model instead of being saved in a third-party application which is still better than nothing but not as effective as the proposed way. It will also be faster and easier for the designer to both save and retrieve it. The demonstrator could not support hyperlinks because of a problem of establishing links between a UNIX- and a Windows- environment but this is a feature that will be possible in the near future and will most likely add more value like links to simulation reports etc.

\section{EVALUATION}

The purpose of the evaluation was to get feedback from the designers on the new method more than to measure the time saved. Feedback like what they thought about working this way, what kind of knowledge and information they feel is necessary to conserve and any other changes they want done.

The evaluation was made at VCC and started with a two hour presentation of the method. After that an individual evaluation was made with six designers that all had some experience with CATIAv5 so that the focus would be on the new method instead of CATIAv5 itself. The evaluation was divided in three phases and with the same six designers in all phases.

In the first phase the designers got to answer different questions using an old CAD-model and all other applications they felt they needed to answer the questions. The model was of a component they never worked with before so they had no knowledge at all about its different features. The questions was typically "Why is that tolerance not standard, what is the function of that hole".

In the second phase the designers got to answer the same questions as in phase one. This time they had access to the new demonstrator model instead and with a little help, since it was a new tool for the designers, they got used to it really fast and had no problems to find the appropriate information in the 3Dannotations.

The third phase consisted of answering a questionnaire with questions about the demonstrator model and the new method in general. Some answers from the designers on the question "Will the work be more effective with the new method?”:

- "Yes, especially when I receive a model or when I hand one over."

- "Yes, an easy way to make links to my own additional information and I can add simulation results directly into the model on to the areas of interest."

- "Yes, very comfortable to have everything in the $3 D$ model instead of the information being spread out on the $2 D$-drawing or in another application. Especially when I want to look at details on previous models which are always done in the conceptual phase." 
During phase one the most common answer about where to get this information was to ask the designer that had created the model. This might work if the designer is still within the company and remembers it all but will be more time consuming for the new designer to find him and get time to ask him and maybe even impossible. But what if the designer isn't within the company or if he has forgotten those details that resulted in that particular design? This might lead to a design that is non optimal, regarding design, weight, performance, and manufacturing cost.

A lot of new areas of use also came up during the evaluation. Some of the participating designers had already their own way to store the design rationale or other knowledge about specific models. Some of them used hand written paper notes that they saved in covers and some of them saved it in text files on their computer. All participants of the evaluation study perceived that the new method was a more efficient way to save the design rationale. They found the idea to receive appropriate and relevant knowledge tightly saved in the model as appealing.

\section{CONCLUSIONS}

Managing this knowledge and information will be more time consuming during the modeling work but the quality will be higher since the designer can keep track of the requirements that's active for the component. The total time will be shorter as well if you consider the whole lifetime of a component. The component may be used first in one project typically spending over three years and may also be used in a later project as a carry-over or by another company in the enterprise. Regarding the two latter examples it's invaluable to have all this knowledge and information tightly integrated with the model and not stored in another application, in paper format or worst case only in the memory of the designer.

The feedback from designers at VCC, both before the demonstrator was built and during the evaluation, clearly states that this is a need and a problem that has to be solved.

The demonstrator that was built along with the evaluation made at VCC clearly shows that it is possible to save and present knowledge and information about components and subsystems in an easy and effective way. A new and larger, more participating designers and longer in time, evaluation is about to take place at VCC as well to cover some areas that the first relatively small evaluation might have missed.

Advantages with this demonstrator and method to work is that it's integrated into the designers everyday CAD-program. This means that the designer doesn't need to work with another program to handle the saving of knowledge and information and it also secures that it will follow with the model and not get lost in another application due to broken links etc. This new method will most likely change the way that VCC has their design review meetings as well. With this new method they can do it all in CATIAv5 and don't have to keep important information about the component saved in a separate document.

One important issue to keep in mind is that the annotations made preferably include date and name of the designer that made them or some other information so it will be traceable. This must be done to keep the information from being redundant or out of date according to new requirements and specifications regarding manufacturing methods etc.

Conclusions based on the evaluation and continuous discussions made with designers at VCC shows that this new method has great potential. If being used to its full potential most of the knowledge and information, design rationale, will be saved within the company instead of being bound to the designer in person. This will ensure that designers will be able to make better informed decisions earlier in the product development process.

Future work in this area will be a study of information and knowledge exchange between VCC and a supplier. The study will include measures of savings in design iterations coupled to the added information and knowledge.

\section{ACKNOWLEDGMENTS}

The authors would like to thank the Swedish Foundation for Strategic Research for the financial support through the ProViking programme. The authors would also like to thank Volvo Cars Corporation for letting us do the case study and evaluation in an industrial environment and also Volvo Information Technology for their help and support. 


\section{REFERENCES}

[1] http://www.asme.org

[2] Isaksson O., A Generative Modeling Approach To Engineering Design, INTERNATIONAL CONFERENCE ON ENGINEERING DESIGN ICED 03, Stockholm, August 19-21, 2003

[3] Bylund, Nicklas., Isaksson, Ola., Kalhori, Vahid., Larsson, Tobias. Enhanced Engineering Design Practice using Knowledge Enabled Engineering with Simulation Methods, 2004 | Conference paper | Design 2004, Dubrovnik, Croatia, May 18-20, 2004

[4] Bylund N., ADRIAN: A software for computing the stiffness of joints and its application in product development 2005 | Journal paper | Journal of Computation in Science and Engineering, Vol 5, No 4, December 2005, JCISE-2004-49.

[5] Sandberg S., Kalhori V., Larsson T., FEM Simulation supported KEE in high strength steel car body design, Proceedings of, IMECE04 2004 ASME International Mechanical Engineering Congress, November 13-20, 2004, Anaheim, California USA

[6] Chapman C.B, Pinfold M., Design engineering-a need to rethink the solution using knowledge based engineering, Knowledge-Based Systems 12 (1999) 257-267

[7] Jungk, R. and Müllert, N. (1987) "Future workshops: How to create desirable futures" London: Institute for Social Inventions

[8] Kensing, F. \& Madsen, K.H., Generating Visions: Future Workshops and Metaphorical Design. In Greenbaum, J. \& Kyng, M., Design at work: Cooperative design of computer systems. (London: Lawrence Erlbaum Associates, 1991) 